\title{
Large Gate Modulation in the Current of a Room Temperature Single Molecule Transistor
}

\author{
Bingqian $\mathrm{Xu}^{1}$, Xiaoyin $\mathrm{Xiao}^{1}$, Xiaomei Yang ${ }^{2}$, Ling Zang*,2 and Nongjian Tao*, ${ }^{1}$ \\ ${ }^{I}$ The Center for Solid State Electronics Research, Arizona State University, Tempe, AZ85287, \\ ${ }^{2}$ Department of Chemistry and Biochemistry, Southern Illinois University, Carbondale, IL62901
}

\section{Supporting Information}

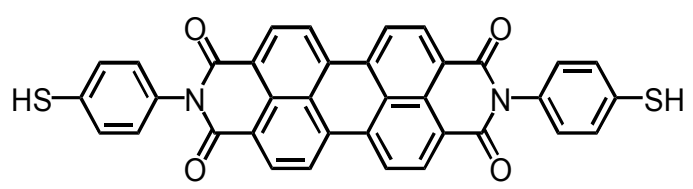

N,N'-di(4-thiophenol)-perylene-3,4,9,10-tetracarboxyl diimide (T-PTCDI)

T-PTCDI was synthesized following the standard condensation methods developed by Langhals. ${ }^{1}$ Briefly, $0.31 \mathrm{~g}(0.78 \mathrm{mmol})$ perylene-3,4,9,10-tetracarboxylic dianhydride (Aldrich), $0.78 \mathrm{~g}$ (6.24 mmol) 4-aminothiophenol (Aldrich), and $6 \mathrm{~g}$ imidazole were heated under argon at $100{ }^{\circ} \mathrm{C}$ for $3 \mathrm{~h}$. The reaction mixture was cooled to room temperature and dispersed in $100 \mathrm{~mL}$ ethanol followed by addition of $300 \mathrm{~mL} 2 \mathrm{M} \mathrm{HCl}$. The mixture was stirred for overnight. The resulting red solid was collected by vacuum filtration through a $0.45 \mu \mathrm{m}$ membrane filter (Osmonics). It was washed thoroughly with distilled water until the $\mathrm{pH}$ of washings turned to be neutral. The collected solid was dried in vacuum at $60{ }^{\circ} \mathrm{C}$. TLC: $\mathrm{R}_{\mathrm{f}}$ (silica $\mathrm{gel} / \mathrm{CHCl}_{3}: \mathrm{methanol}$ $20: 1)=0.85$.

T-PTCDI is slightly soluble in DMF, DMSO, and little less soluble in other solvents like chloroform, toluene, and dioxane. The homogeneous solution of T-PTCDI was prepared by sonicating a certain amount of solid in the solvent, followed by filtration with $0.45 \mu \mathrm{m}$ PTFE membrane filter. Due to the low solubility, no good NMR spectrum could be obtained even with overnight running. Mass spectra were run on a MALDI instrument in the negative mode $(-\mathrm{H})$ with angiotensin as an external standard. MS (M-H), $m / z$ : 605.74 (calc. 605.67).

A single PTCDI molecule was wired to two gold electrodes using a STM break junction technique developed previously in our lab at ASU. ${ }^{2}$ The technique creates individual molecular junctions by repeatedly moving a gold tip (source electrode) into and out of contact with a gold substrate (drain electrode) on which PTCDI molecules are adsorbed from DMSO solutions (Fig. 1a). The process is controlled by a feedback loop that starts by driving the tip into contact with the substrate using a piezoelectric transducer (PZT). Once the contact is fully established, the feedback loop activates the PZT to pull the two electrodes out of contact. After breaking the contact, the molecules can bridge the tip and the substrate to form a molecular junction. Further pulling causes breakdown of the individual molecules from contacting the electrodes, which is shown as a series of steps appeared in the conductance (Fig. 1c). Histograms constructed with $\sim 1000$ of individual measurements show peaks located near integer multiples of a fundamental conductance value, $1.2 \times 10^{-5} \mathrm{G}_{0}$, where $\mathrm{G}_{0}=2 \mathrm{e}^{2} / \mathrm{h} \sim 77 \mu \mathrm{S}$, with e the electron charge and $\mathrm{h}$ the 
Planck constant (Fig. 1d). The lowest peak corresponds to the conductance of a single molecule. The histogram analysis provides an unambiguous determination of single molecule conductance averaged over a large number of measurements.

The single molecule conductance was determined at different gate voltages following the same break-junction technique described above. The measurements were performed in aqueous electrolyte solutions after self-assembling the molecules onto $\mathrm{Au}$ electrodes from DMSO solutions. It was found that a decrease in gate voltage (to more negative values) increased the molecular conductance, while an increase in gate voltage (to more positive values) resulted in little change in molecular conductance (Fig. S1). When the gate voltage $\left(\mathrm{V}_{\mathrm{g}}\right)$ was $0 \mathrm{~V}$ (vs. $\mathrm{Ag} / \mathrm{AgCl}$ ), the conductance of the molecule determined from the conductance histogram was $\sim 1.2 \times 10^{-5} \mathrm{G}_{0}$, virtually the same as that obtained in toluene, which indicates negligible role of the solvent in the conductance of PTCDI. The conductance changed little when increasing $\mathrm{V}_{\mathrm{g}}$ to 0.5 $\mathrm{V}$, beyond which gold electrodes begin to oxidize. Decreasing $\mathrm{V}_{\mathrm{g}}$ to $-0.15 \mathrm{~V}$, however, increased the conductance from $\sim 1.2 \times 10^{-5} \mathrm{G}_{0}$ to $\sim 1.5 \times 10^{-5} \mathrm{G}_{0}$ (Fig. S1a). Further decreasing $\mathrm{V}_{\mathrm{g}}$ to $-0.35 \mathrm{~V}$ increased the conductance to $\sim 3.5 \times 10^{-5} \mathrm{G}_{0}$ (Fig. S1b). At $-0.55 \mathrm{~V}$, the conductance increased to $\sim 4.9 \times 10^{-4} \mathrm{G}_{0}, \sim 40$ times greater than that at $0 \mathrm{~V}$ (Fig. S1c). The electrochemical gate-dependent conductance is plotted as open squares in Fig. 2a. Below $\sim-0.7 \mathrm{~V}$, gold-thiol bonds become increasingly unstable, due to the reductive desorption of thiol molecules from the gold electrodes.
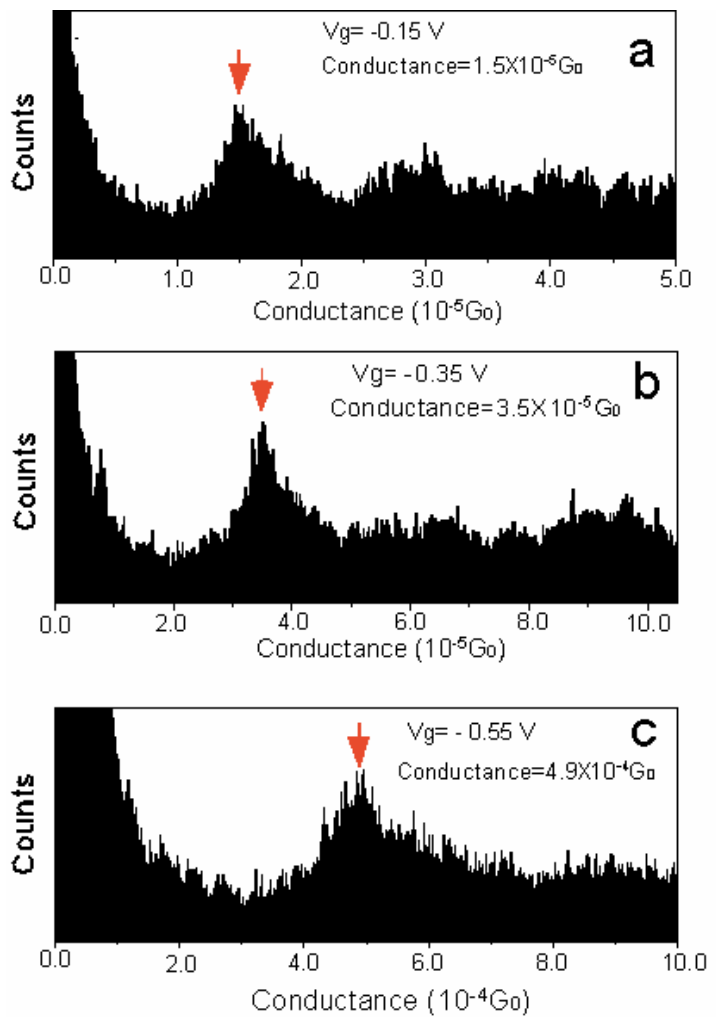

Fig. S1. Conductance histograms of PTCDI molecular wires formed in $0.01 \mathrm{M} \mathrm{NaClO}_{4}$ at various gate voltages. The position of the first peak (pointed by an arrow) gives the average conductance of a single molecule, which shifts with the gate voltage. 
Fig. S2 shows the gate voltage dependence of the $\mathrm{I}_{\mathrm{sd}}-\mathrm{V}_{\mathrm{sd}}$ curve, which is linear within the bias $\left(\mathrm{V}_{\mathrm{sd}}\right)$ range when $\mathrm{V}_{\mathrm{g}}>\sim-0.4 \mathrm{~V}$, but it becomes increasingly nonlinear when $\mathrm{V}_{\mathrm{g}}<\sim-0.4 \mathrm{~V}$. To avoid reductive desorption of thiol at negative potentials and oxidation of gold at positive potentials, the sweep range of $\mathrm{V}_{\mathrm{sd}}$ was adjusted for each $\mathrm{V}_{\mathrm{g}}$ so that both the source and drain gold electrodes were between $-0.6 \mathrm{~V}$ and $+0.4 \mathrm{~V}$.

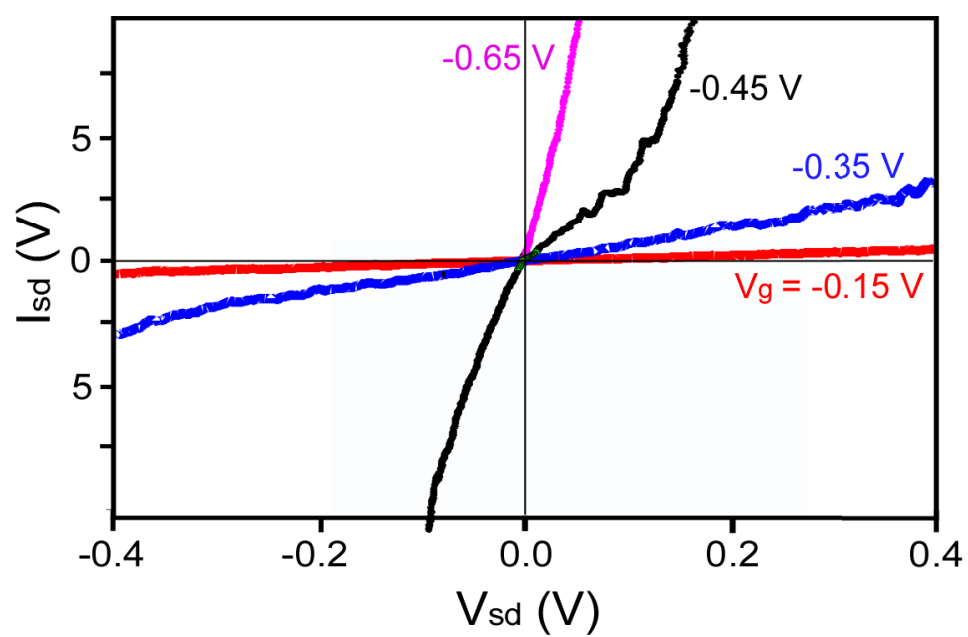

Fig. S2. Linear scale plots of $\mathrm{I}_{\mathrm{sd}}-\mathrm{V}_{\text {sd }}$ curves at various gate voltages. The data are the same as those plotted in Fig. $2 \mathrm{~b}$ in the context.

(1) Langhals, H. Heterocycles 1995, 40, 477-500.

(2) Xu, B. Q.; Tao, N. J. Science 2003, 301, 1221-1223.

\section{Complete Reference 5b:}

Park, J.; Pasupathy, A. N.; Goldsmith, J. L.; Chang, C.; Yaish, Y.; Petta, J. R.; Rinkoski, M.; Sethna, J. P.; Abruna, H. D.; McEuen, P. L.; Ralph, D. C. Nature 2002, 417, 722. 